ORIGINAL ARTICLE

\title{
Violacein antimicrobial activity on Staphylococcus epidermidis and synergistic effect on commercially available antibiotics
}

\author{
H.V. Dodou ${ }^{1}$, A.H. de Morais Batista ${ }^{1}$ (D, G.W.P. Sales ${ }^{1}$, S.C. de Medeiros ${ }^{1}$, M.L. Rodrigues ${ }^{1}$, \\ P.C.N. Nogueira' ${ }^{2}$, E.R. Silveira ${ }^{2}$ and N.A.P. Nogueira ${ }^{1}$ \\ 1 Department of Clinical and Toxicological Analysis, Federal University of Ceará, Fortaleza, Brazi \\ 2 Department of Organic Chemistry, Federal University of Ceará, Fortaleza, Brazil
}

\begin{abstract}
Keywords
antibiotic synergism, antibiotics, antimicrobial activity, Staphylococcus epidermidis, violacein.

\section{Correspondence \\ Hilania Valéria Dodou, Department of Clinical and Toxicological Analysis, Federal University of Ceará, Fortaleza, Ceará, Brazil. \\ E-mail: valeriadodou@gmail.com}

2017/0163: received 20 February 2017, revised 16 June 2017 and accepted 20 July 2017

doi:10.1111/jam.13547

\begin{abstract}
Aims: The study aimed to assess whether violacein has antimicrobial activity on Staphylococcus epidermidis and synergistically modulates the action of commercially available antimicrobial drugs.

Methods and Results: Violacein showed excellent antimicrobial activity on biofilm-forming and nonbiofilm-forming S. epidermidis strains (ATCC 35984) (ATCC 12228), with bacteriostatic $\left(\mathrm{MIC}=20 \mu \mathrm{g} \mathrm{m}^{-1}\right.$ and $10 \mu \mathrm{g} \mathrm{ml}^{-1}$ respectively) and bactericidal effects ( $\mathrm{MBC}=20 \mu \mathrm{g} \mathrm{ml}^{-1}$ for both strains), observed in short periods of exposure. The violacein bactericidal concentration led to $S$. epidermidis death after $2-3 \mathrm{~h}$ of exposure. Additionally, violacein synergistically modulated the activity of different antimicrobial classes on $S$. epidermidis ATCC $12228(81 \cdot 8 \% ; n=9)$ and on S. epidermidis ATCC 35984 $(54 \cdot 5 \% ; n=6)$, reducing the MIC of these antibiotics by up to 16 -fold.

Conclusion: Violacein shows excellent antimicrobial activity on S. epidermidis strains.

Significance and Impact of the Study: Violacein shows the potential for the development of a new drug for the treatment of infections caused by S. epidermidis.
\end{abstract}

\section{Introduction}

Staphylococcus epidermidis is a Gram-positive coccus, which is part of the human skin and mucosal microbiota, although this micro-organism also has pathogenic potential when related to healthcare-associated infections (HAI). It is one of the main infection-causing pathogens associated with medical devices (such as catheters and prostheses, among others) due to virulence factor expression, mainly biofilm formation (Namvar et al. 2014; Chessa et al. 2015).

The emergence of this micro-organism resistant to several antimicrobials in the last decades has become a challenge for treatment choice, as most of the strains isolated in hospital environments are resistant to beta-lactam drugs and cases of strains resistant to glycopeptides have already been described (Montúfar Andrade et al. 2016). Considering this scenario, studies that investigate new effective drugs against this pathogen become very important.
Violacein (VIO) is a metabolite produced by bacteria of different species, especially Chromobacterium violaceum and has several biological activities previously described in the literature, such as its antimicrobial action on Gram-positive bacteria (Choi et al. 2015). Nevertheless, regarding S. epidermidis, the action of this pigment has not been specifically described on this pathogen.

The aim of this study was to evaluate the antimicrobial activity of VIO on biofilm-forming and nonbiofilmforming S. epidermidis and its modulating effect on commercially available antibiotic agents.

\section{Materials and methods}

Violacein (VIO)

The VIO used in this study was extracted from $C$. violaceum ATCC 12472, purified and characterized by 
ultraviolet-visible spectroscopy (modified Rettori and Durán 1998) (Fig. 1). Chromobacterium violaceum ATCC 12472 culture was obtained in nutrient broth and, subsequently, bacterial cells were separated from the culture medium by centrifugation. To extract the violacein, the supernatant was discarded, the bacterial pellet was washed with distilled water three times and submitted to successive extractions with methanol, until the absence of the pigment's characteristic violet colour was attained. This solution was filtered, the methanol was rotavaporated and the methanolic dry extract was obtained, which was then placed in a separation funnel to start the purification process. The separation was performed using methanol, distilled water and ethyl acetate. The hydroalcoholic fraction was collected and submitted to the separation process several times, whereas the violacein-ethyl acetate fraction was collected, dried with sodium sulphate, rotavaporated and kept at $60^{\circ} \mathrm{C}$ for 3 days. The yield was $48 \cdot 86 \%$.

\section{Bacterial strains}

The tested strains were S. epidermidis ATCC 12228 (nonbiofilm forming) and S. epidermidis ATCC 35984 (biofilm forming), obtained from the Collection of Reference Microorganisms on Health Surveillance (Coleção de Microrganismos de Referência em Vigilância Sanitária-CMRVS), FIOCRUZ-INCQS, Rio de Janeiro.
Determination of minimum inhibitory concentration (MIC) and minimum bactericidal concentration (MBC)

The MIC of VIO and the clinically used antibiotics (ATBs) were determined using the microdilution method in culture broth (CLSI 2012). In 96-well, sterile microplates, $100 \mu \mathrm{l}$ of Brain Heart Infusion (BHI) broth (Merck Millipore Corporation), $20 \mu \mathrm{l}$ of different concentrations of VIO $\left(0.019\right.$ to $\left.40 \mu \mathrm{g} \mathrm{ml}^{-1}\right)$ or ATBs (0.024 to $\left.50 \mu \mathrm{g} \mathrm{ml}^{-1}\right)$ and $80 \mu \mathrm{l}$ of microbial suspension $\left(10^{6}\right.$

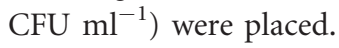

Controls were carried out with sterile BHI broth and BHI broth with microbial inoculum. The microplates were incubated for $24 \mathrm{~h}$ at $37^{\circ} \mathrm{C}$ and the MIC was considered as the lowest concentration of VIO or ATB capable of inhibiting microbial growth.

Inoculums of $5 \mu \mathrm{l}$ from the microplate wells used for MIC determination that showed no visible microbial growth, were plated. The plates were incubated at $37^{\circ} \mathrm{C}$ and the colonies were counted after $24 \mathrm{~h}$. MBC was considered the lowest VIO concentration capable of causing $99.9 \%$ of cell death in relation to the initial inoculum (Shanholtzer et al. 1984). The assays were performed in triplicate.

Determination of the effect of violacein exposure time on microbial viability-Time-kill assay

Aliquots of $20 \mu \mathrm{l}$ of $\mathrm{VIO}$, at concentrations equal to $1 / 2$ MIC, MIC, $2 \times$ MIC and $4 \times$ MIC, were added to

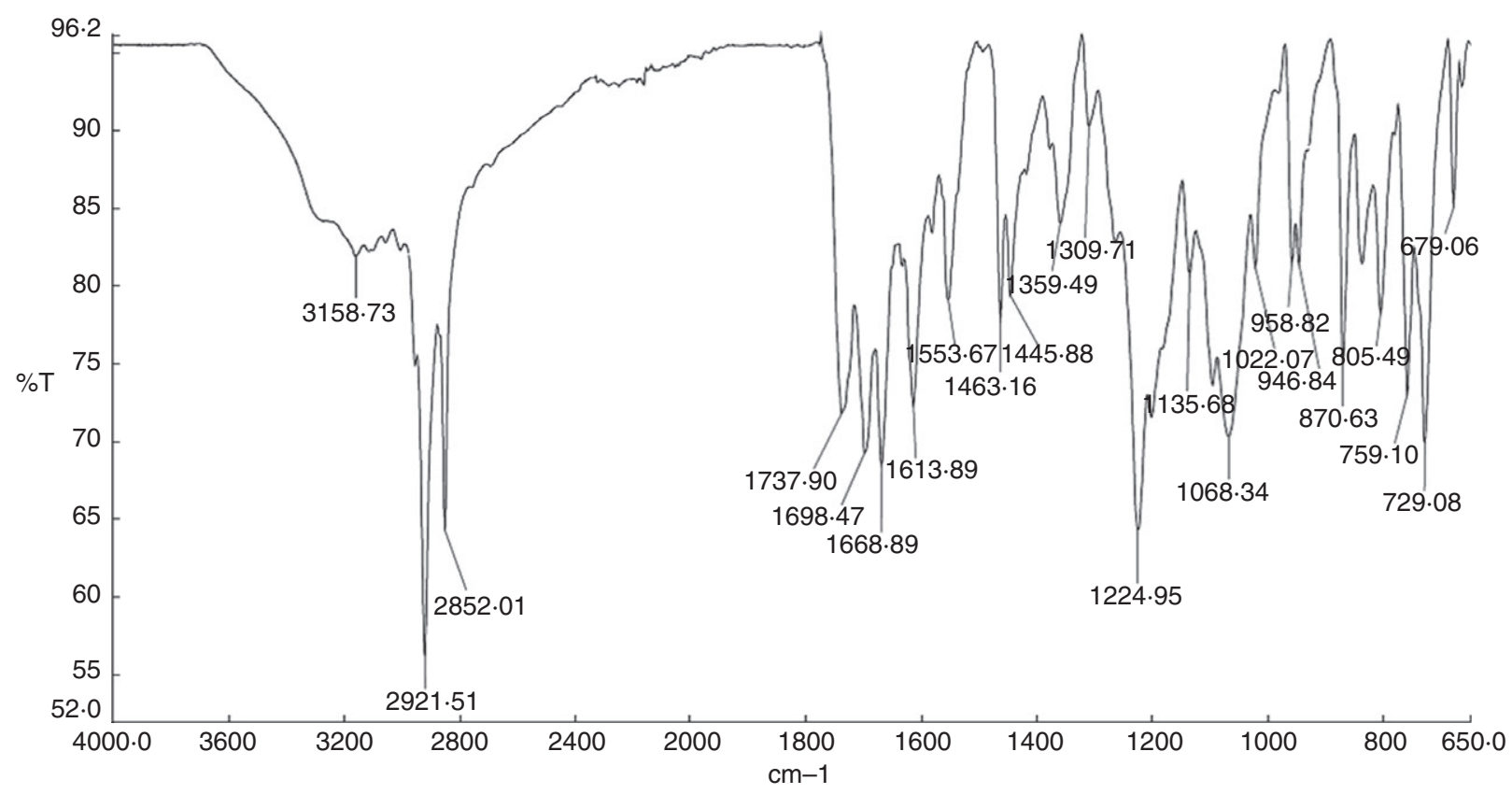

Figure 1 Absorption spectrum in the VIO infrared region. 
96-well microplates containing $100 \mu \mathrm{l}$ of BHI broth and $80 \mu \mathrm{l}$ of the microbial suspension $\left(10^{6} \mathrm{CFU} \mathrm{ml}{ }^{-1}\right)$. The microplates were incubated at $37^{\circ} \mathrm{C}$ and $5 \mu$ l aliquots were removed, diluted in sterile $0.85 \%$ saline solution and plated at times zero and every $30 \mathrm{~min}$ until $6 \mathrm{~h}$ of incubation. The counting of the colonies that grew on the plates was performed after $24 \mathrm{~h}$ of incubation at $37^{\circ} \mathrm{C}$ (Olajuyigbe and Afolayan 2012). Microbial cultures not treated with VIO were used as experimental controls.

\section{Determination of VIO modulatory action on antibiotics} (ATB)

The effect of VIO on the activity of the clinically used ATBs, such as piperacillin + tazobactam, amikacin, gentamicin, ciprofloxacin, cefepime, ceftazidime, vancomycin, meropenem, ceftriaxone, cephalothin and oxacillin, was evaluated by the checkerboard method (Cleeland and Squires 1991). In 96-well microplates, aliquots of $80 \mu \mathrm{l}$ of microbial cultures $\left(10^{6} \mathrm{CFU} \mathrm{ml}{ }^{-1}\right)$, $80 \mu \mathrm{l}$ of BHI broth, $20 \mu \mathrm{l}$ of VIO and $20 \mu \mathrm{l}$ of ATB were added, at the final concentrations of $1 / 2,1 / 4,1 / 8$ and $1 /$ $16 \times$ MIC. The microplates were incubated for $24 \mathrm{~h}$ at $37^{\circ} \mathrm{C}$ and visual inspection of the microbial growth was performed.

To evaluate the effect of the associations, the fraction inhibitory concentration index (FICI) (Shin and Lim 2004) was calculated: $\mathrm{FICI}=\mathrm{FIC}_{\mathrm{VIO}}+\mathrm{FIC}_{\mathrm{ATB}}=[\mathrm{VIO}] /$ $\mathrm{MIC}_{\mathrm{VIO}}+[\mathrm{ATB}] / \mathrm{MIC}_{\mathrm{ATB}}$. Where, $[\mathrm{VIO}]$ is the lowest concentration of VIO in association with antimicrobial activity; $\mathrm{MIC}_{\mathrm{VIO}}$ is the MIC of VIO alone; $\mathrm{FIC}_{\mathrm{VIO}}$ is the Fraction Inhibitory Concentration of VIO; [ATB] is the lowest concentration of ATB in association with antimicrobial activity; $\mathrm{MIC}_{\mathrm{ATB}}$ is the $\mathrm{MIC}$ of $\mathrm{ATB}$; $\mathrm{FIC}_{\mathrm{ATB}}$ is the Fractional Inhibitory Concentration of ATB. The FIC Index (FICI) was interpreted as: synergistic effect for FICI $\leq 0 \cdot 5$, additive or indifferent effect for FICI $>0.5$ and $<1 \cdot 0$, and antagonistic effect for FICI $>1 \cdot 0$ (Eucast 2003).

\section{Determination of the effect of VIO-ATB association exposure time on microbial viability - Time-kill assay}

To perform this assay, two synergistic associations were chosen, with the lowest concentrations of VIO for each micro-organism found in the checkerboard test. Twentymicrolitre aliquots of VIO and ATB, alone and in combination, were added to the wells of the 96-well microplate containing $80 \mu \mathrm{l}$ of BHI broth and $80 \mu \mathrm{l}$ of the microbial suspensions $\left(10^{6} \mathrm{CFU} \mathrm{ml} \mathrm{m}^{-1}\right)$. The microplates were incubated at $37^{\circ} \mathrm{C}$ and at time intervals of $0,2,4,6,8,10,12$ and $24 \mathrm{~h}, 5-\mu \mathrm{l}$ aliquots were removed, diluted in $0.85 \%$ sterile saline and plated. After $24 \mathrm{~h}$ of incubation at $37^{\circ} \mathrm{C}$, colonies that had grown on the medium surface were counted (Olajuyigbe and Afolayan 2012).

\section{Statistical analysis}

Statistical analysis was performed using ANOVA with Dunnett's post-test. The assays were performed in triplicate and the results expressed as mean $\pm \mathrm{SD}$ and were considered significant when $P<0 \cdot 05$.

\section{Results}

\section{VIO antimicrobial activity}

VIO showed excellent antimicrobial, bacteriostatic and bactericidal activities on the tested S. epidermidis strains, as shown in Table 1.

In the time-kill assay, when strains were exposed to $1 / 2$ VIO MIC, growth inhibition was observed after $5 \mathrm{~h}$ and $6 \mathrm{~h}$ for S. epidermidis ATCC 12228 and S. epidermidis ATCC 35984 respectively. When exposed to VIO MIC, growth inhibition was observed for S. epidermidis ATCC 12228 and loss of viability after $3 \mathrm{~h}$ for S. epidermidis ATCC 35984. When exposed to concentrations equal to $2 \times$ MIC and $4 \times$ MIC of VIO, there was loss of cell viability after $2 \mathrm{~h}$ and $1 \mathrm{~h}$ for S. epidermidis ATCC 12228, respectively, and after $2 \mathrm{~h} 30 \mathrm{~min}$ and $1 \mathrm{~h} 30 \mathrm{~min}$ for $S$. epidermidis ATCC 35984 respectively (Fig. 2).

VIO MIC $\left(10 \mu \mathrm{g} \mathrm{ml}^{-1}\right)$ for S. epidermidis ATCC 12228 was capable of reducing cell growth by $33.3 \%$ in the first two hours of incubation. For S. epidermidis ATCC 35984, VIO MIC $\left(20 \mu \mathrm{g} \mathrm{ml}^{-1}\right)$ reduced microbial growth by $40 \cdot 7 \%$, also after $2 \mathrm{~h}$ of incubation.

\section{VIO modulatory activity}

Modulatory activity assays showed a high synergistic effect between VIO and most of the tested antimicrobials used in clinical practice. For S. epidermidis ATCC 12228, VIO showed a synergistic and additive effect on $81.8 \%$ $(n=9)$ and $18 \cdot 2 \%(n=2)$ of the tested ATBs, respectively, with no antagonistic effect in any VIO-ATB

Table 1 Minimum Inhibitory Concentration (MIC) and Minimum Bactericidal Concentration (MBC) of VIO for Staphylococcus epidermidis strains

\begin{tabular}{lll}
\hline Microbial strains & $\mathrm{MIC}\left(\mu \mathrm{g} \mathrm{ml}^{-1}\right)$ & $\mathrm{MBC}\left(\mu \mathrm{g} \mathrm{ml}^{-1}\right)$ \\
\hline Staphylococcus epidermidis & 10 & 20 \\
ATCC 12228 & & \\
Staphylococcus epidermidis & 20 & 20 \\
ATCC 35984 & & \\
\hline
\end{tabular}


S. epidermidis ATCC 12228

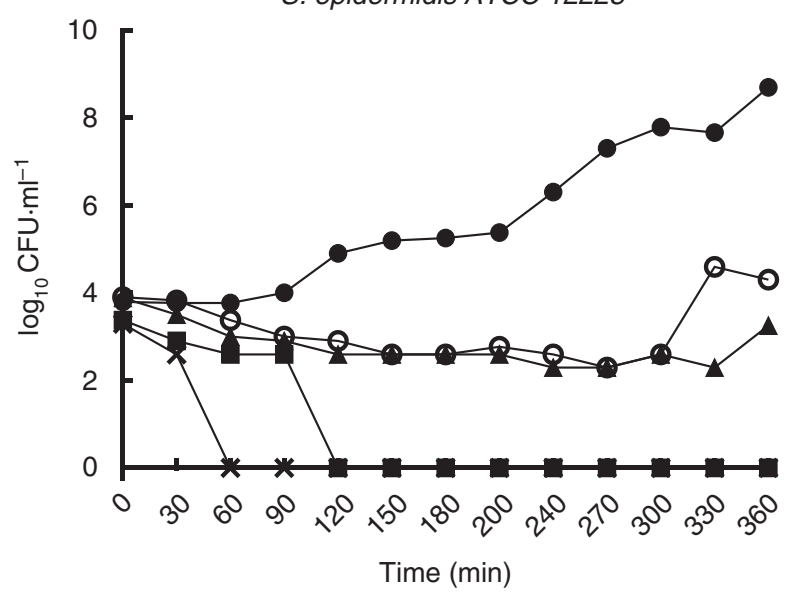

S. epidermidis ATCC 35984

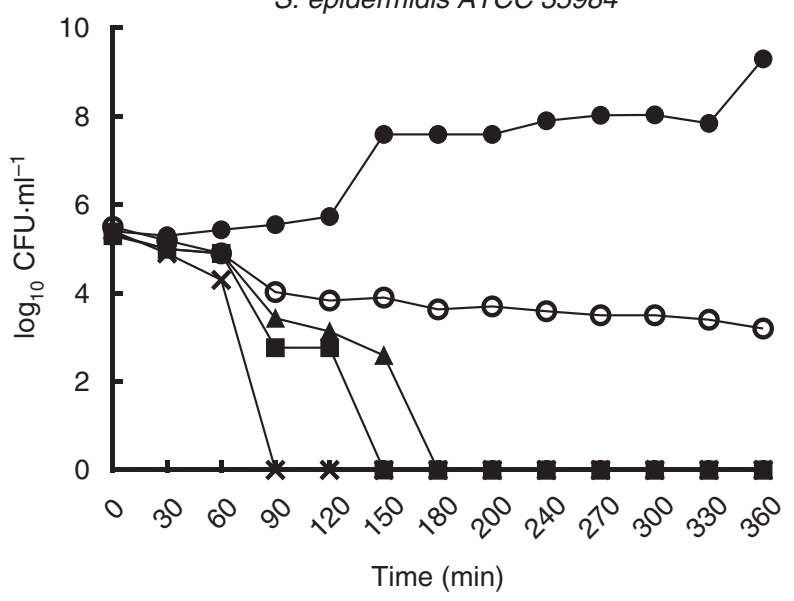

Figure 2 Effect of exposure time at different VIO concentrations on viability of Staphylococcus epidermidis. (-) Control: without VIO; (O) 1/2

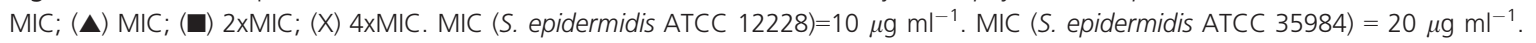

association. In the associations that showed a synergistic effect, VIO was capable of reducing the MIC by 16 -fold of five ATBs (55.5\%): gentamicin, ceftazidime, vancomycin, cephalothin and oxacillin; it also reduced the MIC by eightfold of one ATB (11.1\%): meropenem; and reduced the MIC by fourfold of three ATBs (33.3\%): amikacin, cefepime and ceftriaxone (Table 2).

For S. epidermidis ATCC 35984, there was a synergistic and additive effect in $54.5 \%(n=6)$ and $18 \cdot 2 \%(n=2)$ respectively. There was no growth inhibition for the other associations between VIO and the ATBs piperacillin + tazobactam, amikacin and gentamicin $(27.3 \% ; n=3)$. VIO reduced the MIC by 16-fold of the six drugs that it showed synergism with: ciprofloxacin, cefepime, ceftazidime, vancomycin, meropenem and cephalothin (Table 3).

In the cell-death assay of the VIO-ATB association, it was demonstrated that all combinations inhibited microbial growth over the entire period (24 h), when compared to the control and the substances alone (Figs 3 and 4).

\section{Discussion}

VIO showed excellent antimicrobial activity, with bacteriostatic and bactericidal actions, on biofilm-forming (ATCC 12228) and nonbiofilm-forming S. epidermidis (ATCC 35984), and synergistically modulated the effect of several clinically used antimicrobials, reducing up to 16-fold the concentrations required to inhibit microbial growth.

The literature reports an important in vitro antimicrobial activity of VIO on Gram-positive micro-organisms, especially on Staphylococcus aureus; however, in relation to Gram-negative bacteria, VIO shows less effect on most species (Durán et al. 2012).

One study demonstrated VIO action on $S$. aureus, with a MIC of $7.8 \mu \mathrm{g} \mathrm{ml}^{-1}$ and $15.6 \mu \mathrm{g} \mathrm{ml}^{-1}$ for methicillinsensitive and resistant strains, respectively, whereas for Gram-negative bacteria, the observed MIC was much higher, $125 \mu \mathrm{g} \mathrm{ml}^{-1}$ for Pseudomonas aeruginosa and $1000 \mu \mathrm{g} \mathrm{ml}^{-1}$ for Klebsiella pneumoniae (Aruldass et al. 2015).

In the present study, we verified that VIO showed excellent antimicrobial action against the Gram-positive pathogen, S. epidermidis, as it showed bacteriostatic and bactericidal action even at low concentrations.

The minimum concentration required to inhibit the micro-organism growth was lower for $S$. epidermidis strain ATCC $12228\left(\mathrm{MIC}=10 \mu \mathrm{g} \mathrm{ml}^{-1}\right)$ when compared to S. epidermidis ATCC $35984 \quad\left(\mathrm{MIC}=20 \mu \mathrm{g} \mathrm{ml}^{-1}\right)$, which can be attributed to the biofilm-forming capacity of the latter. For biofilm formation, the bacterium undergoes adaptations in its entire genome, which makes it 10to 1000 -fold more resistant, and justifies antimicrobial substance activity limitations on such micro-organisms (Aguila-Arcos et al. 2015).

The bactericidal and bacteriostatic activities of substances can be determined by time-kill assays, which identify the antimicrobial action velocity of the substance being tested. The cell-death assay showed that VIO has a high antimicrobial effect on both strains of $S$. epidermidis, with a reduction of at least 3 logs in microbial growth when compared to the control. Moreover, its bactericidal action on the tested strains occurred with short exposure times.

The bacteriostatic action was also observed at subinhibitory concentrations $(1 / 2 \times \mathrm{MIC})$ for periods of $5 \mathrm{~h}$ and $6 \mathrm{~h}$ for $S$. epidermidis ATCC 12228 and 
Table 2 Modulatory effect of VIO on the antimicrobial activity of ATBs of clinical use on the growth of Staphylococcus epidermidis ATCC $12228^{*}$

\begin{tabular}{|c|c|c|c|c|c|c|c|c|}
\hline Antibiotics & {$[\mathrm{VIO}] \S$} & $\mathrm{MIC} \mathrm{VIO \dagger}$ & $\mathrm{FIC} \mathrm{VIO**}$ & {$[\mathrm{ATB}] \boldsymbol{\Phi}$} & MIC ATB $\ddagger$ & FIC ATB十† & $\mathrm{FICl}+\mathrm{t}$ & Effect§§ \\
\hline Piperacillin + Tazobactam & 0.625 & 10 & 0.0625 & 1.5625 & $3 \cdot 125$ & 0.5 & 0.5625 & Additive \\
\hline Amikacin & 0.625 & 10 & 0.0625 & 0.7812 & $3 \cdot 125$ & 0.25 & 0.3125 & Synergic \\
\hline Gentamicin & 1.25 & 10 & 0.125 & 0.0244 & 0.3906 & 0.0625 & 0.1875 & Synergic \\
\hline Ciprofloxacin & $2 \cdot 5$ & 10 & 0.25 & 0.0976 & 0.1953 & 0.5 & 0.75 & Additive \\
\hline Cefepime & 0.625 & 10 & 0.0625 & 0.3906 & 1.5625 & 0.25 & 0.3125 & Synergic \\
\hline Ceftazidime & $1 \cdot 25$ & 10 & 0.125 & 0.7812 & $12 \cdot 5$ & 0.0625 & 0.1875 & Synergic \\
\hline Vancomycin & 1.25 & 10 & 0.125 & 0.1953 & 3.125 & 0.0625 & 0.1875 & Synergic \\
\hline Meropenem & 0.625 & 10 & 0.0625 & 0.7812 & $6 \cdot 25$ & 0.125 & 0.1875 & Synergic \\
\hline Ceftriaxone & 0.625 & 10 & 0.0625 & 1.5625 & $6 \cdot 25$ & 0.25 & 0.3125 & Synergic \\
\hline Cephalothin & 1.25 & 10 & 0.125 & 0.0122 & 0.1953 & 0.0625 & 0.1875 & Synergic \\
\hline Oxacillin & 1.25 & 10 & 0.125 & 0.0244 & 0.3906 & 0.0625 & 0.1875 & Synergic \\
\hline
\end{tabular}

*The table shows only the association of the minor concentrations in which there was inhibition of microbial growth.

$\dagger \mathrm{MIC}$ VIO: MIC of isolated violacein $\left(\mu \mathrm{g} \cdot \mathrm{ml}^{-1}\right)$.

†MIC ATB: MIC of isolated antibiotics $\left(\mu \mathrm{g} \cdot \mathrm{ml}^{-1}\right)$.

$\S[\mathrm{VIO}]:$ MIB of violacein in association $\left(\mu \mathrm{g} \cdot \mathrm{ml}^{-1}\right)$.

q[ATB]: MIC of antibiotics in association $\left(\mu \mathrm{g} \cdot \mathrm{ml}^{-1}\right)$.

$* * \mathrm{FIC} \mathrm{VIO}=[\mathrm{VIO}] / \mathrm{MIC} \mathrm{VIO}$.

$\dagger \mathrm{H} F \mathrm{C}$ ATB $=[\mathrm{ATB}] / \mathrm{MIC}$ ATB.

$\mathrm{HAFICI}=\mathrm{FIC} \mathrm{VIO} \mathrm{+} \mathrm{FIC} \mathrm{ATB.}$

$\S \S \mathrm{FICl} \leq 0.5$ : synergism; $\mathrm{FICl}>0.5$ and $<1.0$ : additive or indifferent; $\mathrm{FICl} \geq 1.0$ : antagonism (EUCAST 2003).

Table 3 Modulatory effect of VIO in the antimicrobial activity of ATBs of clinical use on the growth of Staphylococcus epidermidis ATCC 35984*.

\begin{tabular}{|c|c|c|c|c|c|c|c|c|}
\hline Antibiotics & {$[\mathrm{VIO}] \S$} & MIC VIO† & $\mathrm{FIC} \mathrm{VIO**}$ & {$[\mathrm{ATB}] \boldsymbol{\Phi}$} & MIC ATB & FIC ATB†† & $\mathrm{FICl}+\mathrm{t}$ & Effect§§ \\
\hline Piperacillin + Tazobactam & $-*$ & 20 & -* & -* & 6.25 & -* & -* & -* \\
\hline Amikacin & $-*$ & 20 & $-*$ & $-*$ & $6 \cdot 25$ & $-*$ & $-*$ & $-*$ \\
\hline Gentamicin & $-{ }^{*}$ & 20 & $-*$ & $-*$ & $12 \cdot 5$ & $-\star$ & $-*$ & $-{ }^{*}$ \\
\hline Ciprofloxacin & $1 \cdot 25$ & 20 & 0.0625 & 0.3906 & $6 \cdot 25$ & 0.0625 & 0.125 & Synergic \\
\hline Cefepime & 5 & 20 & 0.25 & 0.3906 & $6 \cdot 25$ & 0.0625 & 0.3125 & Synergic \\
\hline Ceftazidime & 5 & 20 & 0.25 & 0.7812 & $12 \cdot 5$ & 0.0625 & 0.3125 & Synergic \\
\hline Vancomycin & 5 & 20 & 0.25 & 0.3906 & $6 \cdot 25$ & 0.0625 & 0.3125 & Synergic \\
\hline Meropenem & $1 \cdot 25$ & 20 & 0.0625 & 0.7812 & $12 \cdot 5$ & 0.0625 & 0.125 & Synergic \\
\hline Ceftriaxone & 2.5 & 20 & 0.125 & $6 \cdot 25$ & $12 \cdot 5$ & 0.5 & 0.625 & Additive \\
\hline Cephalothin & 5 & 20 & 0.25 & 0.0244 & 0.3906 & 0.0625 & 0.3125 & Synergic \\
\hline Oxacillin & 5 & 20 & 0.25 & 0.7812 & 1.5625 & 0.5 & 0.75 & Additive \\
\hline
\end{tabular}

*The table shows the association of the minor concentrations in which there was inhibition of the microbial growth.

†MIC VIO: MIC of isolated violacein $\left(\mu \mathrm{g} \mathrm{ml}^{-1}\right)$.

\$MIC ATB: MIC of isolated antibiotics $\left(\mu \mathrm{g} \mathrm{ml}^{-1}\right)$.

$\S\left[\right.$ VIO]: MIC of violacein in association $\left(\mu \mathrm{g} \mathrm{ml}^{-1}\right)$.

q[ATB]: MIC of antibiotics in association $\left(\mu \mathrm{g} \mathrm{ml}^{-1}\right)$.

**FIC VIO $=[\mathrm{VIO}] / \mathrm{MIC} \mathrm{VIO}$.

$\dagger \mathrm{FIC}$ ATB $=[\mathrm{ATB}] / \mathrm{MIC}$ ATB.

$\$ \mathrm{FICl}=\mathrm{FIC} \mathrm{VIO}+\mathrm{FIC} \mathrm{ATB}$

$\S \S \mathrm{FICl} \leq 0.5$ : synergism; $\mathrm{FICl}>0.5$ and $<1.0$ : additive or indifferent; $\mathrm{FICl} \geq 1.0$ : antagonism (Eucast 2003).

-*There was no growth inhibition in none of the associations.

S. epidermidis ATCC 35984 respectively. Additionally, VIO bactericidal action verified at $2 \mathrm{~h}$ for $S$. epidermidis ATCC 12228 and at $3 \mathrm{~h}$ for S. epidermidis ATCC 35984, occurred more rapidly when the concentration increased and, therefore, the bactericidal effect of this pigment is concentration dependent.

The increasing resistance of $S$. epidermidis to several antimicrobial drugs (Bender et al. 2015; O'Connor et al. 

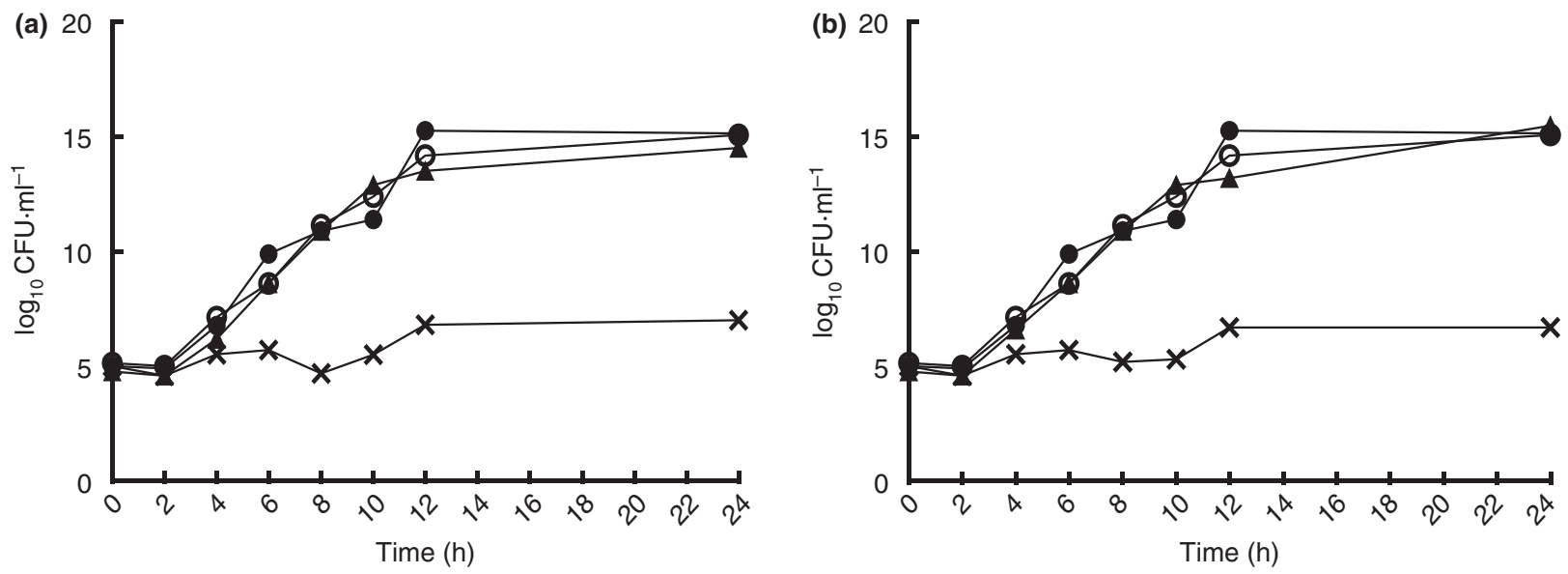

Figure 3 Effect of exposure time to VIO-CEF and VIO-MER associations on viability of Staphylococcus epidermidis ATCC 12228. (a) (-) Control: without VIO and without ATB; (O) VIO $\left(1 / 16 \mathrm{MIC}=0,625 \mu \mathrm{g} \mathrm{ml}^{-1}\right)$; $(\mathbf{\Delta}) \operatorname{CEF}\left(1 / 4 \mathrm{MIC}=0,3906 \mu \mathrm{g} \mathrm{ml}^{-1}\right)$; (X) VIO-CEF. (b) () Control: without VIO and without ATB; (O) VIO $\left(1 / 16 \mathrm{MIC}=0,625 \mu \mathrm{g} \mathrm{ml}^{-1}\right) ;(\mathbf{\Lambda}) \operatorname{MER}\left(1 / 8 \mathrm{MIC}=0,7812 \mu \mathrm{g} \mathrm{ml}^{-1}\right) ;(X)$ VIO-MER.
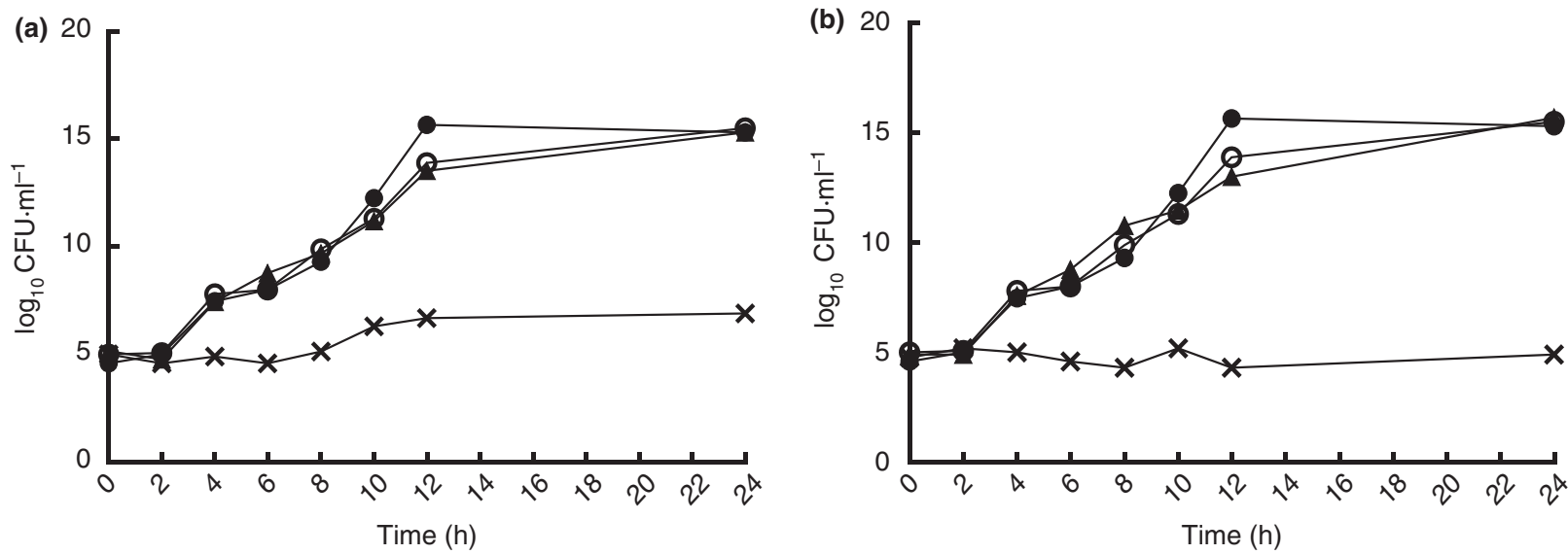

Figure 4 Effect of exposure time to VIO-CIP and VIO-MER associations on viability of Staphylococcus epidermidis ATCC 35984. (a) (•) Control: without VIO and without ATB; (O) VIO $\left(1 / 16 \mathrm{MIC}=1,25 \mu \mathrm{g} \mathrm{ml}^{-1}\right)$; $(\mathbf{\Delta}) \mathrm{CIP}\left(1 / 16 \mathrm{MIC}=0,3906 \mu \mathrm{g} \mathrm{ml}^{-1}\right)$; (X) VIO-CIP. (b) () Control: without VIO and without ATB; (O) VIO (1/16 MIC = 1,25 $\left.\mu \mathrm{g} \mathrm{ml}^{-1}\right) ;(\mathbf{\Lambda}) \operatorname{MER}\left(1 / 16 \mathrm{MIC}=0,7812 \mu \mathrm{g} \mathrm{ml}^{-1}\right)$; (X) VIO-MER.

2015; and Montúfar Andrade et al. 2016) is an important factor to be considered for studies that investigate not only the antibacterial action of new molecules but also evaluate the capacity of such molecules to potentiate the action of commercially available antimicrobial drugs.

Studies show that VIO interacts synergistically with several commercially available antibiotics on Salmonella typhi, Vibrio cholera, P. aeruginosa, K. pneumonia and S. aureus, and with azole antifungals (ketoconazole, fluconazole, clotrimazole and itraconazole) on the dermatophyte fungus Trichophyton rubrum (Subramaniam et al. 2014; Anju et al. 2015).

The present study showed that VIO synergistically modulates the action of several clinically used antimicrobials on biofilm-forming and nonbiofilm-forming S. epidermidis, increasing the effectiveness of antimicrobials of different classes, such as penicillins, aminoglycosides, cephalosporins, glycopeptides and carbapenems for S. epidermidis ATCC 12228; and of quinolones, cephalosporins, glycopeptides and carbapenems for S. epidermidis ATCC 35984.

The time-kill assay can also be performed to determine synergistic combinations between substances, confirming the results of the checkerboard test, as well as identifying the velocity of the associations' bactericidal action. The bacterial mortality rate is identified by counting viable cells (CFU ml ${ }^{-1}$ ) at different times and synergism is considered when a reduction $\geq 2 \log _{10} \mathrm{CFU} \mathrm{ml^{-1 }}$ occurs due to exposure to the association, when compared to the inhibition caused by the substances alone (Jayaraman et al. 2010). 
When assessing the influence of time of exposure on the association activity, two associations were selected for each one of the strains (VIO-CEF and VIO-MER for $S$. epidermidis ATCC 12228; and VIO-CIP and VIO-MER for S. epidermidis ATCC 35984). All tested associations were capable of significantly inhibiting microbial growth for up to $24 \mathrm{~h}$ when compared to the inhibition resulting from the isolated use of the substances, at the same concentrations used in the association. These results demonstrate that VIO is highly capable of potentiating the effect of commercially available antibiotics.

Considering the increasing rates of bacterial resistance to multiple drugs, synergistic combinations between new molecules and commercially available drugs may be a good alternative to control such micro-organisms, due to factors such as prevention of the emergence of resistant strains and toxicity reduction, since the same effect is obtained with much lower concentrations, in addition to the fact that the action spectrum will be increased in relation to that observed for the substances alone.

The present study demonstrates the excellent antimicrobial activity of VIO on S. epidermidis, with a high synergistic modulating effect when associated with clinically used antimicrobials. Since there are no studies that described the action of VIO specifically on S. epidermidis, this is a pioneering study that can be used as the basis for new researches investigating the action mechanism of this pigment on S. epidermidis.

\section{Acknowledgements}

We acknowledge Coordenação de Aperfeiçoamento de Pessoal de Nível Superior (CAPES) for the financial support and the Federal University of Ceará for the structure and financial support.

\section{Conflict of Interest}

The authors declare no conflicts of interest.

\section{References}

Aguila-Arcos, S., Ding, D., Aloria, K., Arizmendi, J.M., Fearnley, I.M., Walker, J.E., Goni, F.M. and Alkorta, I. (2015) A commensal strain of Staphylococcus epidermidis overexpresses membrane proteins associated with pathogenesis when grown in biofilms. J Membrane Biol, 248, 431-442.

Anju, S., Kumar, N. S., Krishnakumar, B. and Kumar, B. S. D. (2015). Synergistic combination of violacein and azoles that leads to enhanced killing of major human pathogenic dermatophytic fungi Trichophyton rubrum. Front Cell Infect Microbiol, 5, 57-68.
Aruldass, C. A., Rubiyatno, R., Venil, C. K. and Ahmad, W. A. (2015) Violet pigment production from liquid pineapple waste by Chromobacterium violaceum UTM5 and evaluation of its bioactivity. RSC Adv, 5, 51524-51536.

Bender, J., Strommenger, B., Steglich, M., Zimmermann, O., Fenner, I., Lensing, C., Dagwadordsch, U., Kekulé, A.S. et al. (2015) Linezolid resistance in clinical isolates of Staphylococcus epidermidis from German hospitals and characterization of two cfr-carrying plasmids. J Antimicrob Chemother 70, 1630-1638.

Chessa, D., Ganau, G. and Mazzarello, V. (2015) An overview of Staphylococcus epidermidis and Staphylococcus aureus with a focus on developing countries. J Infect Dev Ctries 9, 547-550.

Choi, S. Y., Yoon, K., Lee, J. I. and Mitchell, R. J. (2015) Violacein: Properties and production of a versatile bacterial pigment. Biomed Res Int, 2015, 465056-465063.

Cleeland, R. and Squires, E. (1991) Evaluation of new antimicrobials in vitro and in experimental animal infections. Antibiotics Lab Med, 3, 739-787.

CLSI. (2012). Methods for dilution antimicrobial susceptibility tests for bacteria that grow aerobically: Approved standard. Clinical and Laboratory Standards Institute. Available at https://clsi.org/standards/products/microbiology/docume $\mathrm{nts} /$ ?page $=1 \&$ sort $=$ code\&sortdir $=$ asc .

Durán, M., Ponezi, A.N., Faljoni-Alario, A., Teixeira, M.F.S., Justo, G.Z. and Durán, N. (2012) Potential applications of violacein: A microbial pigment. Med Chem Res, 21, $1524-1532$.

EUCAST (2003) Determination of minimum inhibitory concentrations (MICs) of antibacterial agentes by broth diluition. Clin Microbiol J, 9, 1-7.

Jayaraman, P., Sakharkar, M.K., Lim, C.S., Tang, T.H. and Sakharkar, K.R. (2010) Activity and interactions of antibiotic and phytochemical combinations against Pseudomonas aeruginosa in vitro. Int J Biol Sci, 6, 556-568.

Montúfar Andrade, F.E., Muñoz, C.A.M., Franco, J.P.V., Correa, L.M.D., Rivera, J.D.V., Miranda, J.V., Londoño, A.M.B., Tobón, J.J.Z. et al. (2016) Bacteremia por Staphylococcus coagulasa negativo com concentración inhibitoria mínima para vancomicina $\geq 2$. Infectio, 20, 3-8.

Namvar, A. E., Bastarahang, S., Abbasi, N., Ghehi, G. S., Farhadbakthiarian, S., Arezi, P., Hosseini, M., Baravati, S. Z. et al. (2014) Clinical characteristics of Staphylococcus epidermidis: A systematic review. Hyg Infect Control, 9, 1811-1820.

O'Connor, C., Powell, J., Finnegan, C., O’Gorman, A., Barrett, S., Hopkins, K.L., Pichon, B., Hill, R. et al. (2015) Incidence, management and outcomes of the first cfrmediated linezolid-resistant Staphylococcus epidermidis outbreak in a tertiary referral centre in the Republic of Ireland. J Hosp Infect, 90, 316-321.

Olajuyigbe, O.O. and Afolayan, A.J. (2012) In vitro antibacterial and time-kill assessment of crude methanolic stem bark extract of Acacia mearnsii De Wild against bacteria in shigellosis. Molecules, 17, 2103-2118. 
Rettori, D. and Durán, N. (1998) Production, extraction and purification of violacein: An antibiotic pigment produced by Chromobacterium violaceum. World J Microbiol Biotechnol, 14, 685-688.

Shanholtzer, C.J., Peterson, L.R., Mohn, M.L., Moody, J.A. and Gerding, D.N. (1984) MBCs for Staphylococcus aureus as determined by macrodilution and microdilution techniques. Antimicrob Agents Chemother, 26, 214-219.
Shin, S. and Lim, S. (2004) Antifungal effects of herbal essential oils alone and in combination with ketoconazole against Trichophyton spp. J Appl Microbiol 97, 1289-1296.

Subramaniam, S., Ravi, V. and Sivasubramanian, A. (2014) Synergistic antimicrobial profiling of violacein with comercial antibiotics against pathogenic micro-organisms. Pharm Biol, 52, 86-90. 